\title{
The role of gut microbiota in the pathogenesis and treatment of acute pancreatitis: a narrative review
}

\author{
Wei-Wei Lu ${ }^{1,2 \#}$, Xi Chen ${ }^{2 \#}$, Jian-Li Ni ${ }^{3,4 \#}$, Sheng-Liang Zhu ${ }^{1}$, Ai-Hua Fei ${ }^{2}$, Xiao-Su Wang ${ }^{1}$ \\ ${ }^{1}$ Department of Gastroenterology, Yueyang Hospital of Integrated Traditional Chinese and Western Medicine, Shanghai University of Traditional \\ Chinese Medicine, Shanghai, China; ${ }^{2}$ Department of Emergency, Xinhua Hospital, School of Medicine, Shanghai Jiao Tong University, Shanghai, \\ China; ${ }^{3}$ Department of Traditional Chinese Medicine of Xinhua Hospital, Shanghai Jiao Tong University School of Medicine, Shanghai, China; \\ ${ }^{4}$ Department of Traditional Chinese Medicine, Chongming Branch Hospital Affiliated to Xinhua Hospital, Shanghai Jiao Tong University School of \\ Medicine, Shanghai, China \\ Contributions: (I) Conception and design: WW Lu, X Chen, JL Ni, XS Wang; (II) Administrative support: SL Zhu, AH Fei, XS Wang; (III) Provision \\ of study materials or patients: WW Lu, X Chen; (IV) Collection and assembly of data: X Chen, JL Ni; (V) Data analysis and interpretation: WW Lu, \\ AH Fei, XS Wang; (VI) Manuscript writing: All authors; (VII) Final approval of manuscript: All authors. \\ \#These authors contributed equally to this work. \\ Correspondence to: Xiao-Su Wang. Department of Gastroenterology, Yueyang Hospital of Integrated Traditional Chinese and Western Medicine, \\ Shanghai University of Traditional Chinese Medicine, No. 110, Ganhe Road, Shanghai 200437, China, Email: xswangxs0084@163.com; Ai-Hua \\ Fei. Department of Emergency, Xinhua Hospital, School of Medicine, Shanghai Jiao Tong University, No. 1665, Kongjiang Road, Shanghai 200092, \\ China. Email: feiaihua@xinhuamed.com.cn.
}

\begin{abstract}
To investigate the role played by gut microbiota in the development and treatment of acute pancreatitis. Gut microbiota is the largest micro-ecosystem in the human body, and is related to various system diseases. Acute pancreatitis is one of the common acute critical diseases in clinical practice, and there are various causative factors for the occurrence of this disease, such as alcohol, infection, obstruction and intestinal microecological factors. The dysbiosis of gut microbiota may play an important role in the pathogenesis of acute pancreatitis and affect prognoses, including gut microbiota structure disorder and bacterial translocation. It can also affect host metabolism and increase the production of toxic metabolites and affect the treatment of acute pancreatitis. Probiotics are live microorganisms that can give health benefits to the host when applied in sufficient quantities, which can effectively stimulate the growth and reproduction of the normal flora of the body, inhibit the overgrowth of pathogenic bacteria, and have a protective effect on the intestinal barrier function. A search of electronic databases (PubMed, EMBASE, Cochrane) has been realized to summarize the information. The paper briefly describes the concept of gut microbiota and acute pancreatitis, examines the role of gut microbiota in the development and treatment of acute pancreatitis, concludes the investigations of the therapeutic effect of probiotics for dysbiosis of gut microbiota in acute pancreatitis in order to provide a valid reference for the development of subsequent clinical strategies.
\end{abstract}

Keywords: Gut microbiota; acute pancreatitis; pathogenesis; treatment

Submitted Jan 21, 2021. Accepted for publication Mar 20, 2021.

doi: $10.21037 /$ apm-21-429

View this article at: http://dx.doi.org/10.21037/apm-21-429

\section{Introduction}

\section{Gut microbiota and acute pancreatitis}

\section{Gut microbiota}

The human intestinal tract is inhabited by more than 500 species of bacteria with a population of up to 100 trillion, weighing about 1,271 grams. The common gut bacteria in the human body are mainly composed of 9 phyla, in which $0.1 \%$ are facultative anaerobic bacteria dominated by enterobacteria, and $99.9 \%$ are obligate anaerobic bacteria 
dominated by bacillus and bifidobacterial. There are more than 1,500 species of bacteria in the human intestinal tract, consisting of more than 50 classes, of which Firmicutes is the most prevalent, followed by Bacteroidetes; other common species include Proteobacteria and Actinobacteria, Fusobacteria, and Verrucomicrobia $(1,2)$.

Gut microbiota has the characteristics of being dynamic and large in quantity, variety, complexity. Gut microbiota includes beneficial bacteria, intermediate bacteria or conditionally pathogenic bacteria, and harmful bacteria. The interdependence and antagonism between the three, and the maintenance of a balanced and stable intestinal environment are the focus of clinical treatments (3). The gut microbiota has the following physiological functions: (I) it provides a protective effect by serving as a barrier for the host. Specifically, after the colony is nascent, it prevents harmful and pathogenic bacteria from colonizing in the intestinal tract, and prevents and defends against foreign infections; (II) it promotes intestinal peristalsis, aids in digestion, and facilitates the body's absorption of nutrients; (III) the intestinal bacteria can produce enzymes, such as $\beta$-glucuronidase, sulfatase, which aid in the metabolism of endogenous proteins, and can be used indirectly or directly by the host; (IV) specific bacteria, such as Bifidobacterium and Lactobacillus, can scavenge free radicals and act as anti-aging agents; and $(\mathrm{V})$ certain intestinal flora, such as anaerobic Corynebacterium, can activate immune cells in the body, improve phagocytosis, regulate immune function, and have anti-cancer effects (4).

The human intestinal barrier system comprises the intestinal flora, the intestinal mucosal immune system, and the intestinal mucosal epithelium (5). The stability of the gut microflora depends on the presence of the intestinal barrier function (6). When the intestinal barrier function is intact, it can usually prevent the migration of intestinal bacteria to outside the intestine; however, the impairment of the barrier function causes the migration of intestinal bacteria from the intestine to other parts of the intestine or even to outside the intestine (7).

\section{Acute pancreatitis}

According to the severity of a patient's clinical symptoms, acute pancreatitis can be divided into mild acute pancreatitis for which edema is the main manifestation, and severe acute pancreatitis (SAP) with hemorrhagic necrosis. SAP is a common surgical emergency with rapid onset, progression and severity, and often presents with a systemic inflammatory response and pancreatic necrosis (8). The treatment of acute pancreatitis has progressed with the improvement of various treatment protocols and monitoring measures; however, the mortality rate of SAP is still $10-30 \%$ (9). The common causes of AP are: (I) alcohol, a large amount of alcohol can lead to a sudden increase in pressure in the pancreatic duct and promote the secretion of pancreatic enzymes in large quantities, resulting in the entry of pancreatic enzymes into the interstitium of the pancreas, causing acute pancreatitis. (II) Infection, various bacteria and viruses can cause acute pancreatitis when they enter the pancreatic tissue through blood or lymph. (III) Obstruction, obstruction of the lower bile duct can lead to an increase in pressure in the bile duct, causing bile to flow back into the pancreatic duct, resulting in rupture of the pancreatic vesicles and causing pancreatitis. (IV) The intestinal microecological factors, the intestine is the largest immune organ of the human body, once its dynamic balance of microorganisms is disrupted, it can lead to local and systemic inflammatory reactions in the human body, which will increase the chance of acute pancreatitis (10). The main cause of death in patients with SAP is a systemic inflammatory response caused by infection, which in turn can lead to systemic inflammatory response syndrome (SIRS) and multiple organ failure or multiple organ dysfunction syndrome (MODS), most notably pancreatic necrosis secondary to infection (11).

\section{Associations between gut microbiota and acute pancreatitis}

The inflammatory response in the course of acute pancreatitis can lead to disorders of gut microbiota, bacterial translocation and impaired intestinal barrier function, they are thought to play an important role in the development of complications of AP like pancreatic necrosis and peripancreatic infections $(12,13)$. The intestinal flora is involved in the construction of the intestinal barrier. In acute pancreatitis, the intestinal flora and the intestinal barrier mutually affect each other to participate in the disease progression.

This review summarizes the alterations in intestinal flora during the development, progression and treatment of AP to reveal the mechanisms of intestinal dysfunction in AP development and summarizes the therapeutic role of probiotics, ultimately providing potential therapeutic strategies for AP.

We present the following article in accordance with the Narrative Review reporting checklist (available at http:// 
dx.doi.org/10.21037/apm-21-429).

\section{Methods}

According to the study, the English electronic databases PubMed, EMBASE, and Cochrane were searched for studies and clinical randomized controlled trials (RCTs) related to acute pancreatitis and gut microbiota in the past 10 years, and the results were summarized.

\section{Discussion}

\section{The effects of gut microbiota on the pathogenesis of acute pancreatitis}

A number of studies have shown that the gut microbiota plays an important regulatory role in the development and progression of acute pancreatitis. For example, Li et al. (14) used ribonucleic acid-related molecular techniques to detect peripheral blood in patients with acute pancreatitis and found that intestinal bacterial deoxyribonucleic acid expression could be detected in the peripheral blood of $68.9 \%$ of patients with acute pancreatitis, and that the detection rate of bacteria was proportionally related to the severity of the patient's condition. Studies $(14,15)$ have shown that the pathogenic bacteria from the intestinal tract that are detectable in peripheral blood mainly include Escherichia coli, Enterococcus, Enterobacteria, Acinetobacter, Shigella flexneri, and Bacillus coagulants. Escherichia coli, Enterococci and Enterobacteriaceae are the main pathogenic bacteria of secondary intestinal infections caused by acute pancreatitis.

Tan et al. (16) found that the levels of inflammatory factors, such as interleukin (IL)-1, IL-6 and tumor necrosis factor alpha, in patients with acute pancreatitis were positively correlated with the content of Enterobacteriaceae and Enterococcus in the intestine, and negatively correlated with the content of Bifidobacterium, suggesting that during the development of acute pancreatitis, the dysbiosis of the gastrointestinal tract can contribute to an increase in the severity of the SIRS. Quigley et al. (17) examined the classification of intestinal flora in patients with SAP and found that a proportional imbalance occurred, as evidenced by an increased proportion of intestinal aerobic bacteria dominated by Escherichia coli, while the growth of anaerobic bacteria, such as bifidobacterial, was inhibited and these bacteria were reduced in number.

It has been suggested that intestinal beneficial flora can induce Paneth cells to secrete bacteriocins $(18,19)$, enhance the secretion of nonspecific and specific immunoglobulin A (20), improve intestinal immune function, and protect the intestinal mucosal barrier. The normal intestinal mucosal barrier consists of a mechanical barrier, chemical barrier, immune barrier, and biological barrier. The mechanical barrier is the most important intestinal mucosal barrier, and is formed by intact intestinal mucosal epithelial structures that are tightly interconnected with each other. Studies have shown that gut microbiota can modulate intestinal mucosal barrier function by influencing intestinal epithelial cell regeneration, intestinal permeability, intestinal antimicrobial peptide release, and the intestinal mucosal layer (21).

Patients with acute pancreatitis complicated with infections caused by intestinal bacteria were found to have an increased endotoxin level, and a higher incidence of intra-abdominal hypertension and abdominal compartment syndrome, which leads to multiple organ dysfunction. In severe cases, the condition can be life threatening and can lead to death if left untreated (22). In addition, the therapeutic measures, such as fasting and gastrointestinal decompression, commonly adopted during SAP treatment, and the microcirculatory disorders caused by reduced blood volume, can lead to intestinal ischemia, hypoxia, and weakened intestinal motility, which impairs the normal elimination of harmful substances and pathogenic flora, which in turn can lead to intestinal dysbiosis.

The simultaneous adoption of massive rehydration strategies during treatment can cause intestinal reperfusion, leading to intestinal wall cell dysfunction, increased intestinal permeability, decreased intestinal barrier function, and the disruption of the intestinal microecological balance. This causes intestinal dysbiosis and translocation, increased endotoxin release, and an increased inflammatory response in SAP patients (23). Prolonged fasting and gastrointestinal decompression therapy can also lead to nutritional deficiencies in the intestinal mucosal epithelium, including energy supplementation and glutamine deficiency, which can result in atrophy and even the apoptosis of the intestinal mucosal epithelium, which in turn can result in the thinning of the intestinal villi and further increased intestinal mucosal permeability, making the intestinal mucosal barrier more susceptible to intestinal flora migration and exacerbating the inflammatory response and infections in patients with SAP (24). Similarly, in patients with fasting or total parenteral nutrition, the reduction of chemical digestives, such as gastric acid, 
bile, lysozyme, not only results in the breakdown of the intestinal chemical barrier, but also in the weakening of the chemical bactericidal effect, thus promoting the dominant multiplication of pathogenic bacteria (25).

During the treatment of pancreatitis, broad spectrum antibiotics are often used to control the infection, which leads to the inhibition of the normal flora of the intestinal tract. Due to the lack of the inhibitory effect of the normal flora, the fungi and drug-resistant pathogenic bacteria in the intestinal tract can multiply more easily. An imbalance of the flora categories and proportions disturbs the environmental micro-ecological balance of the intestinal tract. Further, the bacteria in the intestinal tract are more prone to retrograde infection through the bile ducts or pancreatic ducts to the peritoneal cavity, or even through the intestinal barrier system to the outside of the intestine. Thus, maintaining the stability and function of the intestinal barrier is also an important part of the treatment of SAP (26).

\section{The effects of gut microbiota on the treatment of acute pancreatitis}

Antibiotics are often administered prophylactically to patients with SAP to inhibit the overgrowth of harmful intestinal bacteria, to control intestinal flora imbalances, and to prevent complications from secondary SAP infections. However, in recent years, a number of clinical studies and meta-analyses have shown that the prophylactic use of antibiotics does not reduce the risk of infection. Conversely, it has also been shown that long-term use of antibiotics is likely to result in drug-resistant bacteria and fungal infections. Several randomized controlled studies have shown that probiotics is effective in treating a variety of gastrointestinal disorders (27). These studies claim that the application of probiotics can correct intestinal microecological imbalances and maintain intestinal barrier function, thereby reducing endogenous infections and achieving the goal of treating gastrointestinal diseases. However, there is still some controversy about the use of probiotics in the treatment of acute pancreatitis.

\section{Probiotics and their mechanism}

The World Health Organization defines probiotics as live microorganisms that can give the host health benefits (28). A large amount of experimental evidence supports the health benefits of probiotics. Currently, the probiotics commonly used clinically include bifidobacteria, lactic acid bacteria, streptococcus, Escherichia coli, Lactococcus lactis and some enterococci; the only non-pathogenic Saccharomyces boulardii is the yeast used. Clinically, these different types of probiotics are widely used to treat patients with irritable bowel syndrome, inflammatory bowel disease, acute infectious diarrhea and other gastrointestinal system diseases; however, their application in the treatment of acute pancreatitis is still relatively rare.

Antibiotics are drugs that we use to inhibit bacteria, viruses, and parasites. But antibiotics inhibit disease-causing bacteria as well as beneficial flora. And as drugs when antibiotics enter the body, they have a therapeutic effect and also cause many adverse effects. The most obvious is the increase of bacterial resistance. The intake of probiotics, on the other hand, does not need to consider the problem of drug resistance, and basically does not have side effects.

Probiotics have been found to inhibit the overgrowth of harmful intestinal flora, while also repairing the protective intestinal barrier and stimulating the production of antiinflammatory factors, which can be used to treat disease (29). In relation to the main mechanism that achieves this, research has shown the following: (I) probiotics and harmful flora have a competitive effect, inhibiting the growth of pathogenic bacteria. Additionally, the bioactive substances produced by probiotics can inhibit or kill pathogenic bacteria, thus regulating the balance of intestinal flora (30). The organic acids produced by probiotic metabolism can lower the potential of hydrogen of a patient's intestinal tract, impairing the viability of pathogenic bacteria and reducing the release of toxins (31); (II) probiotics stimulate the secretion of mucin from the intestinal mucosa and inhibit the binding of pathogenic bacteria to the intestinal epithelium, reducing their passage through the intestinal wall into peripheral organs (32); (III) probiotics promote the biosynthesis of glutathione, improve intestinal mucosal barrier dysfunction, reduce oxidative stress and reduce ileal mucosal permeability and apoptosis levels, thereby inhibiting intestinal bacterial migration (33); and (IV) probiotics can affect the function of immune cells in a variety of ways (e.g., by regulating the production of proand anti-inflammatory cytokines).

\section{The application of probiotics in the acute pancreatitis animal model}

In recent years, some animal studies have shown that probiotics are effective in the treatment of SAP (33-35). A meta-analysis of probiotic therapy studies performed on animal models of SAP showed that probiotics reduced the incidence of infectious complications, improved pancreatic 
histological scores, and reduced mortality in SAP rats (36). Further, van Minnen et al. (7) found that probiotics reduce the overgrowth of potentially pathogenic bacteria in the duodenum, thereby reducing the migration of intestinal bacteria out of the intestinal lumen, including the pancreas, reducing the complications of infection and mortality from SAP.

\section{The use of probiotics used in clinical trials}

The results of a random clinical trial conducted by Oláh et al. (37) showed that SAP patients in an experimental group who were treated with probiotics had a lower incidence of secondary complications, shorter hospital stays, and were less likely to require surgery than those in the control group. However, in a multicenter, largesample, randomized, double-blind controlled clinical trial in Europe, an opposite conclusion was drawn. This randomized controlled trial by Besselink et al. (38) examined 296 patients with SAP who were randomly allocated to a control group (144 cases) or a probiotic treatment group (152 cases). In addition to the conventional treatment, the two groups were prescribed either doses of multispecies probiotics or a placebo (administered twice daily for 28 days). The incidence of infectious complications of patients within 90 days after discharge was then examined. However, no reduction in the incidence of infectious complications following prophylactic application among patients in the probiotics treatment group was found; rather, an increased risk of death was found.

\section{The safety of probiotic treatments}

At present, there is a certain level of safety in using probiotics in the treatment of SAP. Kotzampassi et al. (39) believe that the theoretical risks of applying probiotics include toxicity, metabolic effects, immune effects, the transduction of resistance genes, and translocation from the intestine. However, the complications in probiotic therapy that have been reported are rare and often only occur in critically ill patients, immunocompromised patients, premature newborns, and organ transplant recipients. Indeed, most studies have shown that probiotics are safe and well tolerated.

\section{Conclusions}

Acute pancreatitis (especially SAP) is a common clinical acute critical illness. It is characterized by an acute onset, rapid progression, high risk, poor prognosis, and high mortality. Appropriate and efficient treatment strategies are key to reducing complications and improving patient outcomes. Numerous studies have confirmed that the occurrence, development, and prediction of acute pancreatitis are closely related to intestinal gut dysbiosis and bacterial translocation. As the intestinal microecosystem is the largest micro-ecosystem in the human body, when it is out of balance, the host's metabolism and the metabolism of its toxic products are affected. A SIRS (including acute pancreatitis) can be easily induced at this time (40). Based on the results of existing animal studies and clinical application trials of probiotic therapies, it appears that probiotics therapy may be a double-edged sword. In the future, further studies need to be conducted, including multicenter, large-scale, high-quality, double-blind randomized controlled studies, that place higher demands on experimental design and sample size. A stratified analysis of the etiology of acute pancreatitis and the imaging factors should also be undertaken to reduce selection bias. The specific types of probiotics used in probiotic therapy, the duration of treatment, the dose of treatment, individual patient differences and human interference factors should also be examined. In conclusion, to date, no consensus on the therapeutic role of probiotics in the treatment of acute pancreatitis has been reached; thus, further research needs to be conducted.

\section{Acknowledgments}

Funding: Shanghai Specialized Research Fund for Integrated Chinese and Western Medicine in General Hospitals (ZHYY-ZXYJHZX-201914).

\section{Footnote}

Reporting Checklist: The authors have completed the Narrative Review reporting checklist. Available at http:// dx.doi.org/10.21037/apm-21-429

Conflicts of Interest: All authors have completed the ICMJE uniform disclosure form (available at http://dx.doi. org/10.21037/apm-21-429). All authors report grants from Shanghai Specialized Research Fund for Integrated Chinese and Western Medicine in General Hospitals, during the conduct of the study. The authors have no other conflicts of interest to declare.

Ethical Statement: The authors are accountable for all 
aspects of the work in ensuring that questions related to the accuracy or integrity of any part of the work are appropriately investigated and resolved.

Open Access Statement: This is an Open Access article distributed in accordance with the Creative Commons Attribution-NonCommercial-NoDerivs 4.0 International License (CC BY-NC-ND 4.0), which permits the noncommercial replication and distribution of the article with the strict proviso that no changes or edits are made and the original work is properly cited (including links to both the formal publication through the relevant DOI and the license). See: https://creativecommons.org/licenses/by-nc-nd/4.0/.

\section{References}

1. Sartor RB. Microbial influences in inflammatory bowel diseases. Gastroenterology 2008;134:577-94.

2. Qin J, Li R, Raes J, et al. A human gut microbial gene catalogue established by metagenomic sequencing. Nature 2010;464:59-65.

3. Farhadi A, Banan A, Fields J, et al. Intestinal barrier: an interface between health and disease. J Gastroenterol Hepatol 2003;18:479-97.

4. Sommer F, Bckhed F. The gut microbiota-masters of host development and physiology. Nat Rev Microbiol 2013;11:227-38.

5. Sand J, Nordback I. Probiotics in severe acute pancreatitis. Lancet 2008;371:634-5.

6. Gou S, Yang Z, Liu T, et al. Use of probiotics in the treatment of severe acute pancreatitis: a systematic review and meta-analysis of randomized controlled trials. Crit Care 2014;18:R57.

7. van Minnen LP, Timmerman HM, Lutgendorff F, et al. Modification of intestinal flora with multispecies probiotics reduces bacterial translocation and improves clinical course in a rat model of acute pancreatitis. Surgery 2007;141:470-80.

8. Banks PA, Bollen TL, Dervenis C, et al. Classification of acute pancreatitis--2012: revision of the Atlanta classification and definitions by international consensus. Gut 2013;62:102-11.

9. Servín-Torres E, Velázquez-García JA, Delgadillo-Teyer Q, et al. Severe acute pancreatitis: surgical management in a third-level hospital. Cir Cir 2009;77:407-10.

10. Maheshwari R, Subramanian RM. Severe Acute Pancreatitis and Necrotizing Pancreatitis. Crit Care Clin 2016;32:279-90.
11. Italian Association for the Study of the Pancreas (AISP), Pezzilli R, Zerbi A, et al. Consensus guidelines on severe acute pancreatitis. Dig Liver Dis 2015;47:532 43.

12. Zhou H, Gao J, Wu WJ, et al. Octreotide ameliorates intestinal dysmotility by interstitial cells of Cajal protection in a rat acute necrotizing pancreatitis model. Pancreas 2011;40:1226-33.

13. Horst NL, Marques RG, Diestel CF, et al. Effects of probiotic supplementation on markers of acute pancreatitis in rats. Curr Ther Res Clin Exp 2009;70:136-48.

14. Li Q, Wang C, Tang C, et al. Bacteremia in patients with acute pancreatitis as revealed by $16 \mathrm{~S}$ ribosomal RNA gene based techniques. Crit Care Med 2013;41:1938-50.

15. Peng JS, Liu ZH, Li CJ, et al: Development of a real-time PCR method for the detection of bacterial colonization in rat models of severe acute pancreatitis. Chin Med J 2010; $123: 326-331$

16. Tan C, Ling Z, Huang Y, et al. Dysbiosis of intestinal microbiota associated with inflammation involved in the progression of acute pancreatitis. Pancreas 2015;44:868-75.

17. Quigley EM, Quera R. Small intestinal bacterial overgrowth: roles of antibiotics, prebiotics, and probiotics. Gastroenterology 2006;130:S78-90.

18. Schlee $M$, Wehkamp J, Altenhoefer A, et al. Induction of human beta-defensin 2 by the probiotic Escherichia coli Nissle 1917 is mediated through flagellin. Infect Immun 2007;75:2399-407.

19. Prescott SL, Wickens K, Westcott L, et al. Probiotic Study Group. Supplementation with Lactobacillus rhamnosus or Bifidobacterium lactis probiotics in pregnancy increases cord blood interferon-gamma and breast milk transforming growth factor-beta and immunoglobin A detection. Clin Exp Allergy 2008;38:1606-14.

20. Odenwald MA, Turner JR. The intestinal epithelial barrier: a therapeutic target? Nat Rev Gastroenterol Hepatol 2017;14:9-21.

21. Singh P, Garg PK. Pathophysiological mechanisms in acute pancreatitis: current understanding. Indian J Gastroenterol 2016;35:153-66.

22. Marotta F, Barreto R, Wu CC, et al. Experimental acute alcohol pancreatitis-related liver damage and endotoxemia: synbiotics but not metronidazole have a protective effect. Chin J Dig Dis 2005;6:193-7.

23. Marik PE, Zaloga GP. Meta-analysis of parenteral nutrition versus enteral nutrition in patients with acute pancreatitis. BMJ 2004;328:1407.

24. Zou XP, Chen M, Wei W, et al. Effects of enteral immunonutrition on the maintenance of gut barrier 
function and immune function in pigs with severe acute pancreatitis. JPEN J Parenter Enteral Nutr 2010;34:554-66.

25. Kecskés G, Belágyi T, Oláh A. Early jejunal nutrition with combined pre- and probiotics in acute pancreatitis-prospective, randomized, double-blind investigations. Magy Seb 2003;56:3-8.

26. Freedman SB, Ali S, Oleszczuk M, et al. Treatment of acute gastroenteritis in children: an overview of systematic reviews of interventions commonly used in developed countries. Evid Based Child Health 2013;8:1123-37.

27. Lin DC. Probiotics as functional foods. Nutr Clin Pract 2003;18:497-506.

28. Sanders ME, Guamer F, Ouerrant R, et al. An update on the use and investigation of probiotics in health and disease. Gut 2013;62:787-96.

29. Rashid SK, Idris-Khodja N, Auger C, et al. Probiotics (VSL\#3) prevent endothelial dysfunction in rats with portal hypertension: role of the angiotensin system. PLoS One 2014;9:e97458. Erratum in: PLoS One 2014;(8):e106467.

30. Park HJ, Lee GH, Jun J, et al. Multiple-unit tablet of probiotic bacteria for improved storage stability, acid tolerability, and in vivo intestinal protective effect. Drug Des Devel Ther 2016;10:1355-64.

31. Sánchez E, Nieto JC, Boullosa A, et al. VSL\#3 probiotic treatment decreases bacterial translocation in rats with carbon tetrachloride induced cirrhosis. Liver Int 2015;35:735-45.

32. Qin RY, Zou SQ, Wu ZD, et al. Influence of splanchnic vascular in fusion on the content of endotoxins in plasma and the translocation of intestinal bacteria in rats with acute hemorrhage necrosis pancreatitis. World J Gastroenterol 2000;6:577-80.

Cite this article as: $\mathrm{Lu} W W$, Chen X, Ni JL, Zhu SL, Fei AH, Wang XS. The role of gut microbiota in the pathogenesis and treatment of acute pancreatitis: a narrative review. Ann Palliat Med 2021;10(3):3445-3451. doi: 10.21037/apm-21-429
33. Muftuoglu MA, Isikgor S, Tosun S, et al. Effects of probiotics on the severity of experimental acute pancreatitis. Eur J Clin Nutr 2006;60:464-8.

34. Lutgendorff F, Trulsson LM, Van Minnen LP, et al. Probiotics enhance pancreatic glutathione biosynthesis and reduce oxidative stress in experimental acute pancreatitis. Am J Physiol Gastrointest Liver Physiol 2008;295:G1111-21.

35. Horst NL.Marques RG, Diestel CF, et al. Effects of probiotic supplementation on markers of acute pancreatitis in rats. Curr Ther Res Clin Exp 2009;70:136-48.

36. Hooijmans CR, de Vries RB, Rovers MM, et al. The effects of probiotic supplementation on experimental acute pancreatitis: a systematic review and meta-analysis. PLoS One 2012;7:e48811.

37. Oláh A, Belágyi T, Pótó L, et al. Synbiotic control of inflammation and infection in severe acute pancreatitis: a prospective, randomized, double blind study. Hepatogastroenterology 2007;54:590-4.

38. Besselink MG, Van Santvoort HC, Buskens E, et al. Probiotic prophylaxis in predicted severe acute pancreatitis: a randomized, double-blind, placebo-controlled trial. Lancet 2008;371:651-9.

39. Kotzampassi K, Giamarellos-Bourboulis EJ. Probiotics for infectious diseases: more drugs, less dietary supplementation. Int J Antimicrob Agents 2012;40:288-96.

40. Nakaharai K, Morita K, Jo T, et al. Early prophylactic antibiotics for severe acute pancreatitis: A populationbased cohort study using a nationwide database in Japan. J Infect Chemother 2018;24:753-8.

(English Language Editor: L. Huleatt) 